\title{
COMMON FIXED POINT THEOREMS WITHOUT CONTINUITY AND COMPATIBILITY IN INTUITIONISTIC FUZZY METRIC SPACE
}

\author{
Jong SEO PARK*
}

\begin{abstract}
In this paper, we prove some common fixed point theorems for finite number of discontinuous, non-compatible mappings on non-complete intuitionistic fuzzy metric spaces and obtain the example. Our research improve, extend and generalize several known results in intuitionistic fuzzy metric spaces.
\end{abstract}

\section{Introduction}

Sessa[12] generalized this content and introduced weakly commuting maps. Also, Jungck and Rhoades[1] introduced the notion of weakly compatible maps and proved that compatible maps are weakly compatible but converse need not true. Many authors have been proved fixed point theorems with conditions of continuity and compatibility in Menger spaces. But Sharma et.al.[13] proved fixed point theorems for non-compatible discontinuous maps in non-complete Menger spaces. Also, Kaleva et.al.[2], Kramosil et.al.[3] have introduced the concept of fuzzy metric space for each different methods, and some authors have been improved generalized and extended several properties in this space.

Recently, Park et.al.[9] defined the intuitionistic fuzzy metric space. Also, Park[6], Park et.al.[10] introduced the notion of compatible maps, and obtained common fixed point theorems in intuitionistic fuzzy metric space.

In this paper, we prove some common fixed point theorems for finite number of discontinuous, non-compatible mappings on noncomplete intuitionistic fuzzy metric spaces and obtain the example. Our research

Received February 17, 2011. Accepted April 12, 2011.

2000 Mathematics Subject Classification. 46S40, 47H10.

Key words and phrases. Non-complete, non-compatible maps, common fixed point. 
improve, extend and generalize several known results in intuitionistic fuzzy metric spaces.

\section{Preliminaries}

We recall some definitions, properties and known results in the intuitionistic fuzzy metric space as following :

Let us recall(see [11]) that a continuous $t$-norm is a operation $*$ : $[0,1] \times[0,1] \rightarrow[0,1]$ which satisfies the following conditions: (a)* is commutative and associative, (b) $*$ is continuous, (c) $a * 1=a$ for all $a \in[0,1],(\mathrm{d}) a * b \leq c * d$ whenever $a \leq c$ and $b \leq d(a, b, c, d \in[0,1])$. Also, a continuous $t$-conorm is a operation $\diamond:[0,1] \times[0,1] \rightarrow[0,1]$ which satisfies the following conditions: $(\mathrm{a}) \diamond$ is commutative and associative, (b) $\diamond$ is continuous, (c) $a \diamond 0=a$ for all $a \in[0,1],(\mathrm{d}) a \diamond b \geq c \diamond d$ whenever $a \leq c$ and $b \leq d(a, b, c, d \in[0,1])$.

Definition 2.1. ([4]) The 5 -tuple $(X, M, N, *, \diamond)$ is said to be an intuitionistic fuzzy metric space if $X$ is an arbitrary set, $*$ is a continuous $t$-norm, $\diamond$ is a continuous $t$-conorm and $M, N$ are fuzzy sets on $X^{2} \times$ $(0, \infty)$ satisfying the following conditions; for all $x, y, z \in X$, such that
(a) $M(x, y, t)>0$,
(b) $M(x, y, t)=1 \Longleftrightarrow x=y$,
(c) $M(x, y, t)=M(y, x, t)$,
(d) $M(x, y, t) * M(y, z, s) \leq M(x, z, t+s)$,
(e) $M(x, y, \cdot):(0, \infty) \rightarrow(0,1]$ is continuous,
(f) $N(x, y, t)>0$,
(g) $N(x, y, t)=0 \Longleftrightarrow x=y$,
(h) $N(x, y, t)=N(y, x, t)$,
(i) $N(x, y, t) \diamond N(y, z, s) \geq N(x, z, t+s)$,
(j) $N(x, y, \cdot):(0, \infty) \rightarrow(0,1]$ is continuous.

Note that $(M, N)$ is called an intuitionistic fuzzy metric on $X$. The functions $M(x, y, t)$ and $N(x, y, t)$ denote the degree of nearness and the degree of non-nearness between $x$ and $y$ with respect to $t$, respectively.

Definition 2.2. ([8]) Let $X$ be an intuitionistic fuzzy metric space.

(a) $\left\{x_{n}\right\}$ is said to be convergent to a point $x \in X$ (written $x_{n} \rightarrow x$ ) if and only if for every $\epsilon>0$ and $\lambda \in(0,1)$, there exists an integer $n_{0}=n_{0}(\epsilon, \lambda)$ such that $M\left(x_{n}, x, \epsilon\right)>1-\lambda, N\left(x_{n}, x, \epsilon\right)<\lambda$ for all $n \geq n_{0}$. 
(b) $\left\{x_{n}\right\}$ is called a Cauchy sequence if for every $\epsilon>0$ and $\lambda \in(0,1)$, there exists an integer $n_{0}=n_{0}(\epsilon, \lambda)$ such that $M\left(x_{n}, x_{n+p}, \epsilon\right)>1-\lambda$, $N\left(x_{n}, x_{m}, \epsilon\right)<\lambda$ for all $n, m \geq n_{0}$.

(c) $X$ is complete if every Cauchy sequence converges in $X$.

Lemma 2.3. ([5])Let $\left\{x_{n}\right\}$ be a sequence in an intuitionistic fuzzy metric space $X$ with $t * t \geq t$ and $t \diamond t \leq t$. If there exist a number $k \in(0,1)$ such that

$$
\begin{aligned}
& M\left(x_{n+2}, x_{n+1}, k t\right) \geq M\left(x_{n+1}, x_{n}, t\right), \\
& N\left(x_{n+2}, x_{n+1}, k t\right) \leq N\left(x_{n+1}, x_{n}, t\right)
\end{aligned}
$$

for all $x, y \in X, t>0$ and $n=1,2, \cdots$, then $\left\{x_{n}\right\}$ is a Cauchy sequence in $X$.

Lemma 2.4. ([7])Let $X$ be an intuitionistic fuzzy metric space. If there exists a number $k \in(0,1)$ such that for all $x, y \in X$ and $t>0$,

$$
M(x, y, k t) \geq M(x, y, t), N(x, y, k t) \leq N(x, y, t),
$$

then $x=y$.

\section{Main Results}

In this section, we introduce the concepts of compatible and weakly compatible maps, and prove the common fixed point theorems for ten mappings satisfying some conditions.

Definition 3.1. ([6])Let $A$ and $B$ be mappings from intuitionistic fuzzy metric space $X$ into itself. The mappings are said to be compatible if

$$
\lim _{n \rightarrow \infty} M\left(A B x_{n}, B A x_{n}, t\right)=1, \quad \lim _{n \rightarrow \infty} N\left(A B x_{n}, B A x_{n}, t\right)=0
$$

for all $t>0$, whenever $\left\{x_{n}\right\} \subset X$ such that $\lim _{n \rightarrow \infty} A x_{n}=\lim _{n \rightarrow \infty} B x_{n}=$ $z$ for some $z \in X$.

Definition 3.2. Let $A$ and $B$ be mappings from intuitionistic fuzzy metric space $X$ into itself. The mappings are said to be weakly compatible if they commute at coincidence point.

Theorem 3.3. Let $A,, B, I, J, L, P, Q, S, T$ and $U$ be self maps on an intuitionistic fuzzy metric space $X$ with $t * t \geq t, t \diamond t \leq t$ for all $t \in[0,1]$ and satisfy the condition:

(a) $P(X) \subset A B I L(X), Q(X) \subset S T J U(X)$, 
(b) There exist $k \in(0,1)$ such that for all $x, y \in X, \alpha \in(0,2)$ and $t>0$,

$$
\begin{aligned}
& M(P x, Q y, k t) \\
& \begin{aligned}
\geq & M(A B I L y, S T J U x, t) * M(P x, S T J U x, t) * M(Q y, A B I L y, t) \\
& * M(Q y, S T J U x, \alpha t) * M(P x, A B I L y,(2-\alpha) t), \\
N(P x, Q y, k t) & \leq\{N(A B I L y, S T J U x, t) \diamond N(P x, S T J U x, t) \diamond N(Q y, A B I L y, t) \\
& \diamond N(Q y, S T J U x, \alpha t) \diamond N(P x, A B I L y,(2-\alpha) t),
\end{aligned}
\end{aligned}
$$

(c)If one of $P(X), A B I L(X), S T J U(X), Q(X)$ is a complete subspace of $X$.

Then (i) $P$ and $S T J U$ have a coincidence point and (ii) $Q$ and $A B I L$ have a coincidence point.

Further if

(d) $A B=B A, A I=I A, A L=L A, B I=I B, B L=L B, I L=L I$, $Q L=L Q, Q I=I Q, Q B=B Q, S T=T S, S J=J S, S U=U S$, $T J=J T, T U=U T, J U=U J, P U=U P, P J=J P$ and $P T=T P$,

(e)The pairs $(P, S T J U)$ and $(Q, A B I L)$ are weakly compatible.

Then (iii) $A, B, I, J, L, P, Q, S, T$ and $U$ have a unique common fixed point in $X$.

Proof. From (a), for any $x_{0} \in X$, there exists $x_{1} \in X$ such that $P x_{0}=A B I L x_{1}$. For such point $x_{1}$, we can choose a point $x_{2} \in X$ such that $Q x_{1}=S T J U x_{2}$ and so on. Inductively, we can construct a sequence $\left\{y_{n}\right\}$ in $X$ such that for $n=0,1,2, \cdots, y_{2 n}=P x_{2 n}=A B I L x_{2 n+1}$ and $y_{2 n+1}=Q x_{2 n+1}=S T J U x_{2 n+2}$.

By (b), for all $t>0$ and $\alpha=1-q$ with $q \in(0,1)$, we have

$$
\begin{aligned}
& \quad M\left(y_{2 n+2}, y_{2 n+1}, k t\right)=M\left(P x_{2 n+2}, Q x_{2 n+1}, k t\right) \\
& \geq M\left(y_{2 n}, y_{2 n+1}, t\right) * M\left(y_{2 n+2}, y_{2 n+1}, t\right) * M\left(y_{2 n+1}, y_{2 n}, t\right) \\
& \quad * M\left(y_{2 n+1}, y_{2 n+1},(1-q) t\right) * M\left(y_{2 n+2}, y_{2 n},(1+q) t\right) \\
& \geq M\left(y_{2 n}, y_{2 n+1}, t\right) * M\left(y_{2 n+2}, y_{2 n+1}, t\right) * M\left(y_{2 n+1}, y_{2 n+2}, q t\right), \\
& N\left(y_{2 n+2}, y_{2 n+1}, k t\right)=N\left(P x_{2 n+2}, Q x_{2 n+1}, k t\right) \\
& \leq N\left(y_{2 n}, y_{2 n+1}, t\right) \diamond N\left(y_{2 n+2}, y_{2 n+1}, t\right) \diamond N\left(y_{2 n+1}, y_{2 n}, t\right) \\
& \quad \diamond N\left(y_{2 n+1}, y_{2 n+1},(1-q) t\right)\left(y_{2 n+2}, y_{2 n},(1+q) t\right) \\
& \leq N\left(y_{2 n}, y_{2 n+1}, t\right) \diamond N\left(y_{2 n+2}, y_{2 n+1}, t\right) \diamond N\left(y_{2 n+1}, y_{2 n+2}, q t\right) .
\end{aligned}
$$


Letting $q \rightarrow 1$, we have

$$
\begin{aligned}
& M\left(y_{2 n+1}, y_{2 n+2}, k t\right) \geq M\left(y_{2 n}, y_{2 n+1}, t\right) * M\left(y_{2 n+1}, y_{2 n+2}, t\right), \\
& N\left(y_{2 n+1}, y_{2 n+2}, k t\right) \leq N\left(y_{2 n}, y_{2 n+1}, t\right) \diamond N\left(y_{2 n+1}, y_{2 n+2}, t\right) .
\end{aligned}
$$

Similarly, we have

$$
\begin{aligned}
& M\left(y_{2 n+2}, y_{2 n+3}, k t\right) \geq M\left(y_{2 n+1}, y_{2 n+2}, t\right) * M\left(y_{2 n+2}, y_{2 n+3}, t\right), \\
& N\left(y_{2 n+2}, y_{2 n+3}, k t\right) \leq N\left(y_{2 n+1}, y_{2 n+2}, t\right) \diamond N\left(y_{2 n+2}, y_{2 n+3}, t\right) .
\end{aligned}
$$

Generally, we have for $m=1,2, \cdots$,

$$
\begin{aligned}
& M\left(y_{m+1}, y_{m+2}, k t\right) \geq M\left(y_{m}, y_{m+1}, t\right) * M\left(y_{m+1}, y_{m+2}, t\right), \\
& N\left(y_{m+1}, y_{m+2}, k t\right) \leq N\left(y_{m}, y_{m+1}, t\right) \diamond N\left(y_{m+1}, y_{m+2}, t\right) .
\end{aligned}
$$

Consequently, for $m=1,2, \cdots$ and $p=1,2, \cdots$, it follows that

$$
\begin{aligned}
& M\left(y_{m+1}, y_{m+2}, k t\right) \geq M\left(y_{m}, y_{m+1}, t\right) * M\left(y_{m+1}, y_{m+2}, \frac{t}{k^{p}}\right), \\
& N\left(y_{m+1}, y_{m+2}, k t\right) \leq N\left(y_{m}, y_{m+1}, t\right) \diamond N\left(y_{m+1}, y_{m+2}, \frac{t}{k^{p}}\right) .
\end{aligned}
$$

Letting $p \rightarrow \infty$, since $M\left(y_{m+1}, y_{m+2}, \frac{t}{k^{p}}\right) \rightarrow 1$ and $N\left(y_{m+1}, y_{m+2}, \frac{t}{k^{p}}\right) \rightarrow$ 0 , we have

$M\left(y_{m+1}, y_{m+2}, k t\right) \geq M\left(y_{m}, y_{m+1}, t\right), \quad N\left(y_{m+1}, y_{m+2}, k t\right) \leq N\left(y_{m}, y_{m+1}, t\right)$.

Hence $\left\{y_{n}\right\}$ is a Cauchy sequence in $X$. Now, suppose that $S T J U(X)$ is complete, then $\left\{y_{2 n+1}\right\} \subset\left\{y_{n}\right\}$ has a limit $z$ in $S T J U(X)$. Let $w \in$ $(S T J U)^{-1} z$. Then $S T J U w=z$.

By (b) with $\alpha=1$, we obtain

$$
\begin{aligned}
& M\left(P w, Q x_{2 n+1}, k t\right)=M\left(P w, y_{2 n+1}, k t\right) \\
& \geq M\left(y_{2 n}, S T J U w, t\right) * M(P w, S T J U w, t) * M\left(y_{2 n+1}, y_{2 n}, t\right) \\
& * M\left(y_{2 n+1}, S T J U w, t\right) * M\left(P w, y_{2 n}, t\right), \\
& N\left(P w, Q x_{2 n+1}, k t\right)=N\left(P w, y_{2 n+1}, k t\right) \\
& \leq N\left(y_{2 n}, S T J U w, t\right) \diamond N(P w, S T J U w, t) \diamond N\left(y_{2 n+1}, y_{2 n}, t\right) \\
& \diamond N\left(y_{2 n+1}, S T J U w, t\right) \diamond N\left(P w, y_{2 n}, t\right) .
\end{aligned}
$$

Letting $n \rightarrow \infty$, we have

$$
M(P w, z, k t) \geq M(P w, z, t), \quad N(P w, z, k t) \leq N(P w, z, t)
$$

Therefore, $P w=z$. Since $S T J U w=z, P w=z=S T J U w$. That is, $w$ is coincidence point of $P$ and $S T J U$. Also, because $P(X) \subset$ 
$A B I L(X), P w=z$ implies that $z \in A B I L(X)$. Let $v \in(A B I L)^{-1} z$, then $A B I L v=z$. By (b) with $\alpha=1$, we have

$$
\begin{aligned}
& M\left(P x_{2 n+2}, Q v, k t\right)=M\left(y_{2 n+2}, Q v, k t\right) \\
& \geq M\left(A B I L v, y_{2 n+1}, t\right) * M\left(y_{2 n+2}, y_{2 n+1}, t\right) * M\left(Q v, y_{2 n+1}, t\right) \\
& \quad * M\left(Q v, y_{2 n+1}, t\right) * M\left(Q x_{2 n+2}, A B I L v, t\right), \\
& N\left(P x_{2 n+2}, Q v, k t\right)=N\left(y_{2 n+2}, Q v, k t\right) \\
& \leq N\left(A B I L v, y_{2 n+1}, t\right) \diamond N\left(y_{2 n+2}, y_{2 n+1}, t\right) \diamond N\left(Q v, y_{2 n+1}, t\right) \\
& \quad \diamond N\left(Q v, y_{2 n+1}, t\right) \diamond N\left(Q x_{2 n+2}, A B I L v, t\right) .
\end{aligned}
$$

Letting $n \rightarrow \infty$, we have

$$
M(Q v, z, k t) \geq M(Q v, z, t), \quad N(Q v, z, k t) \leq N(Q v, z, t) .
$$

Therefore $Q v=z$. Hence $Q v=z=A B I L v$. That is, $v$ is coincidence point of $Q$ and $S T J U$.

If $P(X)$ or $Q(X)$ is complete, then by (a), $z \in P(X) \subset A B I L(X)$ or $z \in Q(X) \subset S T J U(X)$. Thus (i) and (ii) are completely established.

From (e), since $\{P, S T J U\}$ is weakly compatible, therefore $P(S T J U w)=$ $S T J U(P w)$ or $P z=S T J U z$. Also, since $\{Q, A B I L\}$ is weakly compatible, therefore $Q(A B I L v)=(A B I L) Q v$ or $Q z=A B I L z$.

Now, we prove that $P z=z$. By (b) with $\alpha=1$, we obtain

$$
\begin{aligned}
& M\left(P z, Q x_{2 n+1}, k t\right)=M\left(P z, y_{2 n+1}, k t\right) \\
& \geq M\left(y_{2 n}, S T J U z, t\right) * M(P z, S T J U z, t) * M\left(y_{2 n+1}, y_{2 n}, t\right) \\
& \quad * M\left(y_{2 n+1}, S T J U z, t\right) * M\left(P z, y_{2 n}, t\right), \\
& N\left(P z, Q x_{2 n+1}, k t\right)=N\left(P z, y_{2 n+1}, k t\right) \\
& \leq N\left(y_{2 n}, S T J U z, t\right) \diamond N(P z, S T J U z, t) \diamond N\left(y_{2 n+1}, y_{2 n}, t\right) \\
& \diamond N\left(y_{2 n+1}, S T J U z, t\right) \diamond N\left(P z, y_{2 n}, t\right) .
\end{aligned}
$$

Proceeding limit as $n \rightarrow \infty$, we get

$$
M(P z, z, k t) \geq M(P z, z, t), \quad N(P z, z, k t) \leq N(P z, z, t) .
$$

Thus, $P z=z$. Since $S T J U z=P z$, we have $P z=S T J U z=z$. 
By (b) with $\alpha=1$, we have

$$
\begin{aligned}
& M\left(P x_{2 n+2}, Q z, k t\right)=M\left(y_{2 n+2}, Q z, k t\right) \\
& \geq M\left(A B I L z, y_{2 n+1}, t\right) * M\left(y_{2 n+2}, y_{2 n+1}, t\right) * M(Q z, A B I L z, t) \\
& \quad * M\left(Q z, y_{2 n+1}, t\right) * M\left(y_{2 n+2}, A B I L z, t\right), \\
& N\left(P x_{2 n+2}, Q z, k t\right)=N\left(y_{2 n+2}, Q z, k t\right) \\
& \leq N\left(A B I L z, y_{2 n+1}, t\right) \diamond N\left(y_{2 n+2}, y_{2 n+1}, t\right) \diamond N(Q z, A B I L z, t) \\
& \diamond N\left(Q z, y_{2 n+1}, t\right) \diamond N\left(y_{2 n+2}, A B I L z, t\right) .
\end{aligned}
$$

Letting $n \rightarrow \infty$, we have

$$
M(z, Q z, k t) \geq M(z, Q z, t), \quad N(z, Q z, k t) \leq N(z, Q z, t) .
$$

Thus, $Q z=z$. Hence $Q z=A B I L z=z$.

By (b) with $\alpha=1$ and using (d), we get

$$
\begin{aligned}
& M(P z, Q(L z), k t) \\
& \begin{aligned}
\geq & M(A B I L(L z), S T J U z, t) * M(P z, S T J U z, t) * M(Q(L z), A B I L(L z), t) \\
& * M(Q(L z), S T J U z, t) * M(P z, A B I L(L z), t),
\end{aligned} \\
& N(P z, Q(L z), k t) \\
& \leq N(A B I L(L z), S T J U z, t) \diamond N(P z, S T J U z, t) \diamond N(Q(L z), A B I L(L z), t) \\
& \quad \diamond N(Q(L z), S T J U z, t) \diamond N(P z, A B I L(L z), t) .
\end{aligned}
$$

From above results, we have

$$
\begin{aligned}
& M(P z, Q(L z), k t)=M(z, L z, k t) \geq M(z, L z, t), \\
& N(P z, Q(L z), k t)=N(z, L z, k t) \leq N(z, L z, t) .
\end{aligned}
$$

Therefore $L z=z$. Since $A B I L z=z$, we have $A B I z=z$.

Also, by (b) with $\alpha=1$ and using (d), we get

$$
\begin{aligned}
& \quad M(P z, Q(I z), k t) \\
& \geq M(A B I L(I z), S T J U(I z), t) * M(P z, S T J U z, t) * M(Q(I z), A B I L(I z), t) \\
& \quad * M(Q(I z), S T J U z, t) * M(P z, A B I L(I z), t), \\
& N(P z, Q(I z), k t) \\
& \leq N(A B I L(I z), S T J U(I z), t) \diamond N(P z, S T J U z, t) \diamond N(Q(I z), A B I L(I z), t) \\
& \quad \diamond N(Q(I z), S T J U z, t) \diamond N(P z, A B I L(I z), t) .
\end{aligned}
$$

From above results, we have

$$
M(I z, z, k t) \geq M(I z, z, t), \quad N(I z, z, k t) \leq N(I z, z, t) .
$$

Thus $I z=z$. Since $A B I z=z$, we have $A B z=z$. 
Now, we prove that $B z=z$. Put $x=z, y=B z$ in (b) with $\alpha=1$ and using $(\mathrm{d})$, we get

$$
\begin{aligned}
& M(P z, Q(B z), k t) \\
& \begin{aligned}
\geq & M(A B I L(B z), S T J U z, t) * M(P z, S T J U z, t) * M(Q(B z), A B I L(B z), t) \\
& * M(Q(B z), S T J U z, t) * M(P z, A B I L(B z), t),
\end{aligned} \\
& N(P z, Q(B z), k t) \\
& \leq N(A B I L(B z), S T J U z, t) \diamond N(P z, S T J U z, t) \diamond N(Q(B z), A B I L(B z), t) \\
& \quad \diamond N(Q(B z), S T J U z, t) \diamond N(P z, A B I L(B z), t) .
\end{aligned}
$$

Then we have

$$
M(z, B z, k t) \geq M(z, B z, t), \quad N(z, B z, k t) \leq N(z, B z, t) .
$$

Thus $z=B z$. Since $A B z=z$, we have $A z=z=B z$.

We prove that $U z=z$. Put $x=U z, y=z$ in (b) with $\alpha=1$ and using (d), we have

$$
\begin{aligned}
& \quad M(P(U z), Q(z), k t) \\
& \quad \begin{array}{l}
M(A B I L z, S T J U(U z), t) * M(P(U z), S T J U(U z), t) * M(Q z, A B I L z, t) \\
\quad
\end{array} \\
& N(P(U z), Q(z), k t) \\
& \leq N(A B I L z, S T J U(U z), t) \diamond N(P(U z), S T J U(U z), t) \diamond N(Q z, A B I L z, t) \\
& \quad \diamond N(Q z, S T J U(U z), t) \diamond N(P(U z), A B I L z, t) .
\end{aligned}
$$

Then we have

$$
M(U z, z, k t) \geq M(U z, z, t), \quad N(U z, z, k t) \leq N(U z, z, t) .
$$

Thus $U z=z$. Since $S T J U z=z$, we have $S T J z=z$.

In order to prove $J z=z$, we put $x=J z, y=z$ in (b) with $\alpha=1$ and using (d), we have

$$
\begin{aligned}
& M(P(J z), Q(z), k t) \\
& \geq M(A B I L z, S T J U(J z), t) * M(P(J z), S T J U(J z), t) * M(Q z, A B I L z, t) \\
& * M(Q z, S T J U(J z), t) * M(P(J z), A B I L z, t), \\
& N(P(J z), Q(z), k t) \\
& \leq N(A B I L z, S T J U(J z), t) \diamond N(P(J z), S T J U(J z), t) \diamond N(Q z, A B I L z, t) \\
& \diamond N(Q z, S T J U(J z), t) \diamond N(P(U z), A B I L z, t) .
\end{aligned}
$$

Then we have

$$
M(J z, z, k t) \geq M(J z, z, t), \quad N(J z, z, k t) \leq N(J z, z, t) .
$$

Thus $J z=z$. Since $S T J z=z$, we have $S T z=z$. 
To prove $T z=z$ put $x=T z, y=z$ in (b) with $\alpha=1$ and using (d), we have

$$
\begin{aligned}
& \quad M(P(T z), Q(z), k t) \\
& \quad \begin{array}{l}
\quad M(A B I L z, S T J U(T z), t) * M(P(T z), S T J U(T z), t) * M(Q z, A B I L z, t) \\
\quad
\end{array} \quad \begin{array}{l}
N(P(T z), Q(z), k t) \\
\leq N(A B I L z, S T J U(T z), t) \diamond N(P(T z), S T J U(T z), t) \diamond N(Q z, A B I L z, t) \\
\quad \diamond N(Q z, S T J U(T z), t) \diamond N(P(U z), A B I L z, t) .
\end{array}
\end{aligned}
$$

Then we have

$$
M(T z, z, k t) \geq M(T z, z, t), \quad N(T z, z, k t) \leq N(T z, z, t) .
$$

Thus $T z=z$. Since $S T z=z$, we have $S z=z$. Therefore from combining the above results, we get $A z=B z=I z=J z=L z=P z=$ $Q z=S z=T z=U z=z$. That is, $z$ is a common fixed point of $A, B, I, J, L, P, Q, S, T$ and $U$. The uniqueness of common fixed point of this mappings can prove easily from (b).

Example 3.4. Let $(X, d)$ be the metric space with $X=[0,1]$. Denote $a * b=\min \{a, b\}$ and $a \diamond b=\max \{a, b\}$ for all $a, b \in[0,1]$ and let $M_{d}, N_{d}$ be fuzzy sets on $X^{2} \times(0, \infty)$ defined as follows :

$$
M_{d}(x, y, t)=\frac{t}{t+d(x, y)}, \quad N_{d}(x, y, t)=\frac{d(x, y)}{t+d(x, y)} .
$$

Then $\left(M_{d}, N_{d}\right)$ is an intuitionistic fuzzy metric on $X$ and $X$ is an intuitionistic fuzzy metric space. Let self mappings $A, B, I, J, L, P, Q, S, T$ and $U$ be maps from $X$ into itself defined as

$$
\begin{aligned}
& A x=\frac{x}{5}, \quad B x=\frac{x}{3}, \quad I x=x, \quad J x=x, \quad L x=\frac{x}{2} \\
& P x=0, \quad Q x=\frac{x}{6}, \quad S x=x, \quad T x=\frac{x}{2}, \quad U x=\frac{x}{3}
\end{aligned}
$$

for all $x \in X$. Then $P(X)=\{0\} \subset\left[0, \frac{1}{30}\right]=A B I L(X)$ and $Q(X)=$ $\left[0, \frac{1}{6}\right] \subset\left[0, \frac{1}{6}\right]=S T J U(X)$. Clearly, (b),(c),(d) and (e) of Theorem 3.3 are satisfied. Thus all conditions of Theorem 3.3 are satisfied and 0 is a unique common fixed point of $A, B, I, J, L, P, Q, S, T$ and $U$ on intuitionistic fuzzy metric space $X$.

\section{References}

[1] Jungck, G., Rhoades, B.E., 1998. Fixed point for set valued functions without continuity, Ind. J. Pure Appl. Math. 29(3), 227-238. 
[2] Kaleva, O., Seikkala, S., 1984. On fuzzy metric spaces, Fuzzy Sets and Systems $12,215-229$.

[3] Kramosil,J., Michalek J., 1975. Fuzzy metric and statistical metric spaces. Kybernetica 11, 326-334.

[4] Park, J.H., Park, J.S., Kwun, Y.C., 2006. A common fixed point theorem in the intuitionistic fuzzy metric space. Advances in Natural Comput. Data Mining(Proc. 2nd ICNC and 3rd FSKD), 293-300.

[5] Park, J.H., Park, J.S., Kwun, Y.C., 2007. Fixed point theorems in intuitionistic fuzzy metric space(I). JP J. fixed point Theory \& Appl. 2(1), 79-89.

[6] Park, J.S., On some results for five mappings using compatibility of type $(\alpha)$ in a fuzzy metric space, inpress.

[7] Park, J.S., Kim, S.Y., 1999. A fixed point theorem in a fuzzy metric space. F.J.M.S. 1(6), 927-934.

[8] Park, J.S., Kwun, Y.C., 2007. Some fixed point theorems in the intuitionistic fuzzy metric spaces. F.J.M.S. 24(2) 227-239.

[9] Park, J.S., Kwun, Y.C., Park, J.H., 2005. A fixed point theorem in the intuitionistic fuzzy metric spaces. F.J.M.S. 16(2), 137-149.

[10] Park, J.S., Kwun, Y.C., Park, J.H., Some results and example for compatible maps of type $(\beta)$ on the intuitionistic fuzzy metric spaces, inpress.

[11] Schweizer, B., Sklar, A., 1960. Statistical metric spaces. Pacific J. Math. 10, 314-334.

[12] Sessa, S., 1982. On a weak commutativity condition of mappings in fixed point considerations, Publ. Inst. Math. 32(46), 149-153.

[13] Sharma, S., Desphaude, B., Tiwari, R., 2008. Common fixed point theorems for finite number of mappings without continuity and compatibility in Menger spaces, J. Korea Soc. Math. Educ. Ser. B: Pure Appl. Math. 15(2), 135-151.

Jong Seo Park

Department of Mathematics Education, Chinju National University of Education,

Jinju 660-756, Korea.

E-mail: parkjs@cue.ac.kr 